\title{
Stage IVB Paranasal Sinus Cancer AJCC v7
}

National Cancer Institute

\section{Source}

National Cancer Institute. Stage IVB Paranasal Sinus Cancer A/CC v7. NCI Thesaurus.

Code C6071.

Stage IVB includes: (T 4b, Any N, M0); (Any T, N3, M0). T4b: Very advanced local disease.

Tumor invades any of the following: orbital apex, dura, brain, middle cranial fossa, cranial nerves other than maxillary division of trigeminal nerve, nasopharynx, or clivus. N3:

Metastasis in a lymph node, more than $6 \mathrm{~cm}$ in greatest dimension. M0: No distant metastasis. (AJCC 7th ed.) 